\title{
Action-Based Body Maps in the Spinal Cord Emerge from a Transitory Floating Organization
}

\author{
Marcus Granmo, ${ }^{\star}$ Per Petersson, ${ }^{*}$ and Jens Schouenborg \\ Neuronano Research Center, Biomedical Center F10, Lund University, S-221 84 Lund, Sweden
}

\begin{abstract}
During development primary afferents grow into and establish neuronal connections in the spinal cord, thereby forming the basis for how we perceive sensory information and control our movements. In the somatosensory system, myriads of primary afferents, conveying information from different body locations and sensory modalities, get organized in the dorsal horn of the spinal cord so that spinal multisensory circuits receive topographically ordered information. How this intricate pathfinding is brought about during development is, however, largely unknown. Here we show that a body representation closely related to motor patterns emerges from a transitory floating and plastic organization through profound activity-dependent rewiring, involving both sprouting and elimination of afferent connections, and provide evidence for cross-modality interactions in the alignment of the multisensory input. Thus, far from being inborn and stereotypic, the dorsal horn of the spinal cord now appears to be a highly adaptive brain-body interface.
\end{abstract}

Key words: development; somatosensory; structural plasticity; sensorimotor; learning; activity dependent

\section{Introduction}

The primary representation of the body in the dorsal horn of the spinal cord has traditionally been described as a simple map of the skin surface (Molander and Grant, 1985; Brown et al., 1997). However, recent data indicate that this view may not be correct. Instead, termination patterns of different cutaneous fibers in the adult rat lumbar spinal cord appear to be closely related to the locations and receptive fields of the reflex sensorimotor circuits (Levinsson et al., 2002). In the withdrawal reflex circuits, pools of reflex encoding interneurons located in the deep part of the dorsal horn, in turn, are related to the sequence of motor neurons in the ventral horn (Schouenborg et al., 1995). In adult mammals, the nociceptive withdrawal reflex system is functionally organized into modules (Schouenborg, 2003); each module controls a single or a few synergistic muscles and transforms sensory input from a receptive field into appropriate motor activity. The sensitivity distribution with respect to tactile and nociceptive input within the cutaneous excitatory receptive field of each module is an imprint of the withdrawal action of the module (Schouenborg and Weng, 1994). This functionality is achieved through a selforganizing process, termed somatosensory imprinting, which is guided by tactile feedback on spontaneous movements during sleep (Holmberg et al., 1997; Petersson et al., 2003; Waldenstrom et al., 2003) during the first three postnatal weeks, indicating a

Received Feb. 13, 2008; revised April 1, 2008; accepted April 10, 2008.

This work was supported by grants from Swedish Medical Research Council (Project 1013 and Linné Grant), the Medical Faculty of Lund University, Knut and Alice Wallenberg Foundation, Brain Foundation in Sweden, Thorsten and Elsa Segerfalks Foundation, Astrid and Gustaf Kaleen Foundation, and Lars Hierta's Memory.

${ }^{*}$ M.G. and P.P. contributed equally to this work.

Correspondence should be addressed to Marcus Granmo at the above address. E-mail: marcus.granmo@med.lu.se.

DOI:10.1523/JNEUROSCI.0651-08.2008

Copyright $\odot 2008$ Society for Neuroscience $\quad$ 0270-6474/08/285494-10\$15.00/0 high degree of congruity between tactile and nociceptive inputs at some point along the reflex pathways.

Recently, parts of the genetic program underlying differentiation and to some extent the target-finding process with respect to the laminar termination have been described for subgroups of thin and coarse cutaneous afferents and proprioceptors (Chen AI et al., 2006; Chen CL et al., 2006; Kramer et al., 2006). Differences in activity patterns in cells can, in turn, lead to certain changes in gene expression controlling synaptology, growth factors, and transmitters (West, 1999; Poo, 2001; Spitzer, 2002). It is therefore conceivable that the initial molecular guidance of different fiber types create only a crude topography in the dorsal horn that is later modified via experience-dependent processes to reach adult complexity and functionality. However, considering that thin and coarse nerve fibers grow into the dorsal horn at different time points and by different routes (Jackman and Fitzgerald, 2000) and the lack of a clear somatotopic organization in the dorsal root ganglia (Prats-Galino et al., 1999), it is puzzling how the different types of fibers from a given body part are able to locate and make somatotopically appropriate connections in the dorsal horn during development.

We have here mapped the spinal terminations of tactile afferents from different parts of the hindpaw in rats during the early postnatal weeks and compared that with the maturation of the withdrawal reflexes. We also describe how coarse and thin afferent fiber terminations in the dorsal horn get aligned during this time period. Finally, we have studied the role of experiencedependent mechanisms in the developmental sculpturing of these sensorimotor circuits.

\section{Materials and Methods}

Animals used. A total of 150 Wistar [studies on mediolateral (ML)-rostrocaudal (RC) termination patterns] and 180 Sprague Dawley rats (studies on laminar distribution of terminals) of both sexes were used in 
experiments involving histological, behavioral, pharmacological, and electromyographic (EMG) techniques. All animals were kept in a $12 \mathrm{~h}$ day-night cycle at a constant environmental temperature of $21^{\circ} \mathrm{C}, 65 \%$ humidity. Food and water were received ad libitum. All experiments were approved in advance by the Malmö/Lund ethical committee of animal experiments.

Anesthesia. All animals that were subject to surgery or tracer injections were anesthetized with isoflurane gas anesthesia ( $2 \%$ in a mixture of $70 \%$ nitrous oxide and $30 \%$ oxygen). Careful infiltration of the tissues with lidocaine (Xylocaine; $2.0 \mathrm{mg} / \mathrm{ml})$ with adrenaline $(1.2 \mu \mathrm{g} / \mathrm{ml})$ was made before surgery to reduce nociceptive input and to minimize bleeding. Surgery commenced when no movements could be evoked by pinching the skin.

Tracer injections. To study the coarse fiber terminations in the mediolateral-rostrocaudal plane of the dorsal horn during postnatal development, cholera toxin subunit B conjugated to horseradish peroxidase (BHRP) was chosen over cholera toxin subunit B (CTb) because of the much simpler B-HRP protocol, which was important when handling the fragile neonatal spinal cord sections. These tracers have been used to label primary afferent terminals in many studies (Robertson and Arvidsson, 1985; Rivero-Melian and Grant, 1990; Coggeshall et al., 1996; Hughes et al., 2003; Pang et al., 2006). Importantly, B-HRP and CTb both preferentially label coarse fibers (Lindh et al., 1989; Dederen et al., 1994). The similarity in stained termination patterns using the two techniques was confirmed in two postnatal day 21 (P21) animals. To minimize spread of tracer in the periphery, small amounts of B-HRP were injected subcutaneously using a fine glass capillary (tip diameter, $\sim 5 \mu \mathrm{m}$ ) and a Hamilton syringe $7000.5 \mathrm{~N}$ or 7101 . Volumes injected were as follows: at P1 and P8, $0.1 \mu \mathrm{l}$; at P14, $0.2 \mu \mathrm{l}$; adults, $0.5 \mu \mathrm{l}$ per site. Injections were made in both left and right hindpaws. After injection, a very small bolus of tracer was observed subcutaneously. No spreading was seen after retraction of the injection capillary.

To study the coarse and thin fiber terminations in the mediolateraldorsoventral plane of the dorsal horn during postnatal development, the following tracer substances (and concentrations) were used: $1 \%$ wheat germ agglutinin (WGA), $1 \% \mathrm{IB}_{4}$ (isolectin), and $0.1 \% \mathrm{CTb}$ conjugated with different fluorophores (Alexa 488, 555, and 647 conjugates; Invitrogen). P1-P24 pups were used. The volumes of the injections were adjusted to the plantar area of the hindpaw at all ages. To compensate for the growth during the first 24 postnatal days, measurements of hindpaw size were performed in animals taken from three different litters. A clearly linear length growth from $8 \mathrm{~mm}$ at birth with $\sim 1 \mathrm{~mm} / \mathrm{d}$ the first 3 weeks ( $r^{2}=0.99$; length measured from the proximal edge of the heel to the distalmost part of the skin on digit three) was found. The area growth as a function of postnatal age could be expressed as a second-order polynomial equation: $\operatorname{area}(t)=\left(0.19 t^{2}+3.76 t+14.8 ; t=\right.$ postnatal day $)$ with a goodness of fit of $r^{2}=0.97$ (paw areas were measured using hindpaw ink stains). Assuming mainly lateral spread, this relation was used to calculate the peripheral injection volumes of the tracer substances. Epifluorescence microscopy ( $12 \mathrm{~h}$ after injection in 20 rat paws) confirmed that the estimated volumes stained similar fractions of the plantar hindpaw (supplemental Fig. 2, available at www.jneurosci.org as supplemental material).

Perfusion and fixation. In the studies using B-HRP tracer, the rats were deeply anesthetized with sodium pentobarbital 24 (for P2) to 72 (for P21 and adult animals) hours after tracer injections, transcardially perfused, rinsed with PBS (300 mOsm, $\mathrm{pH} 7.4$ ), and fixated with $40-1000 \mathrm{ml}$ of $1 \%$ paraformaldehyde with $1.25 \%$ glutaraldehyde. A dorsal laminectomy was made, and the lumbar spinal cord was removed. The length of the individual spinal cord segments was measured with a micromanipulator, with the caudalmost rootlet defining the caudal border of each segment. The roots were cut, after which the spinal cord was left to dehydrate in $30 \%$ sucrose at $4^{\circ} \mathrm{C}$ overnight.

For horizontal sections, freeze sectioning was used (CM1325; Leica). The slices were cut into $40 \mu \mathrm{m}$ sections and were processed with tetramethyl benzidine (TMB), ammonium heptamolybdate, and $\mathrm{H}_{2} \mathrm{O}_{2}$ in the following sequence [described in detail by Ralston (1994)]: (1) Rinse in buffer containing $\mathrm{NaH}_{2} \mathrm{PO}_{4}$ and $\mathrm{Na}_{2} \mathrm{HPO}_{4}$ on ice. (2) Process in TMB in ethanol mixed with buffer with ammonium heptamolybdate and $\mathrm{H}_{2} \mathrm{O}_{2}$,
$\mathrm{pH} \sim 5$. Incubate for $30 \mathrm{~min}$ to several hours until the desired reaction appears. (3) Rinse in buffer for $10 \mathrm{~min}$.

In the studies using fluorescent tracers, the tracers were allowed to be transported for 1-5 d after injection (depending on whether peripheral nerves, DRGs, or the spinal cord were studied). The animals were anesthetized, and the lumbar part of the spinal column was excised and put into paraformaldehyde (4\%) overnight. Transverse sections of spinal cord and/or DRGs were dissected out of the fixated cord and directly put in an agar block in cold saline. Transverse slices were cut into $100 \mu \mathrm{m}$ sections using a Leica VT1000S microtome, and horizontal sections were cut into $70 \mu \mathrm{m}$ sections using freeze sectioning, as described above.

Microscopy. For B-HRP-labeled slices, the sections were mounted on slides and photographed with a digital microscope camera (DMC; Polaroid) at $40 \times$. For the horizontal sections, dorsal-view maps were constructed (using Photoshop software; Adobe Systems) by superimposing all individual labeled slices from one spinal cord. The scattered terminations generally observed in deeper laminas were not analyzed (Levinsson et al., 2002). To display the relations between labeling and the medial border of the dorsal horn, the latter was approximated to a vertical plane along which horizontal sections were aligned. The maps created for each animal were superimposed within each age group to create an overall picture of the spinal labeling in that age group. The spinal cord termination fields were aligned rostrocaudally to the caudal end of the heel termination, and each pixel was color coded according to the number of animals with labeled terminals in the corresponding area. The mediolateral orientation of the right-side termination (digit 4) was inverted so as to mimic left-side injections. Fluorescent images were collected using a Zeiss LSM 510 confocal microscope. Each fluorophore was scanned separately with appropriate excitation wavelengths and filter settings; no signal overlap was detected between different channels.

Elvax manufacture and implantation. To investigate the involvement of NMDA receptors in the development of the spinal termination pattern of primary afferents, Elvax (ethylene-vinyl acetate copolymer; DuPont) containing either saline $(n=38)$ or MK-801 (noncompetitive NMDA-R antagonist; $n=43$ ) was produced. Elvax was prepared as previously described (Smith et al., 1995), and MK-801 (10 mM) or saline $(0.9 \%)$ was added. The frozen and dried Elvax blocks were cut on a vibratome into 50 $\mu \mathrm{m}$ slices. The slices were then incubated in saline in $37^{\circ} \mathrm{C}$ for $18-24 \mathrm{~h}$ to wash out the initial release of MK-801 reported in previous studies (Smith et al., 1995; Beggs et al., 2002). Six- to seven-day-old rats were anesthetized (as above), and the lumbar spinal cord was exposed at the L4-L5 level. A small incision was made in the dura mater, and the Elvax sections were inserted over the spinal cord. The wounds were sealed, and the rats were returned to their home cage. The animals were regularly controlled after surgery, and if signs of inflammation or motor deficits were evident, the animals were killed.

Neonatal capsaicin treatment. Newborn rat pups received two intraperitoneal injections of $50 \mathrm{mg} / \mathrm{kg}$ (E)-capsaicin (Merck Biosciences), the first a few hours after birth and the second $24 \mathrm{~h}$ later. The injections were made during deep anesthesia ( $5 \%$ isoflurane in a mixture of $65 \%$ nitrous oxide and $35 \%$ oxygen). The capsaicin was dissolved in EtOH to a concentration of $10 \% ; 3 \mu \mathrm{l}$ of the solution was then mixed with $3 \mu$ l of Tween 20 (Sigma) and $144 \mu \mathrm{l}$ of saline to a total volume of $150 \mu \mathrm{l}$, as previously described (Torsney et al., 2000). Behavioral tests commenced a week later.

Chronic vibrations. Six litters $(n=35)$ were placed in a vibrating cage during P1-P24. Gradually increasing time periods in the vibration cage were used to accustom the pups to the conditions and to ascertain a steady weight gain (from $2 \mathrm{~h}$ in the newborn to $20 \mathrm{~h} / \mathrm{d}$ during the third week). Vibrations $(\sim 200 \mathrm{~Hz})$ were generated through the rotation of an eccentrically placed metal wheel underneath the cage. Touching the bottom of the cage gave a tingling sensation in the experimenters similar to touching, for example, a tuning fork.

Surgery and preparation for EMG recordings. The animals were anesthetized and ventilated artificially via a tracheal cannula. The expiratory $\mathrm{CO}_{2}$ level (3.0-4.0\%) was monitored continuously. An infusion of 5\% glucose in Ringer acetate, $\mathrm{pH} 7.0$, at a rate of $10-40 \mu \mathrm{l} / \mathrm{min}$ was administered via the right jugular vein. Mean arterial blood pressure (65-140 $\mathrm{mmHg}$ ) was monitored continuously in the right carotid artery. A lam- 
inectomy of the 10th thoracic vertebra was carried out, and the rat was spinalized using a pair of fine scissors. A craniotomy was performed, and the brain rostral to the inferior colliculus was removed. The anesthesia was then discontinued. Experiments were terminated on signs of deterioration, such as a precipitous drop in blood pressure or in expiratory $\mathrm{CO}_{2}$ level. After termination of the experiments, the animals were given a lethal dose of isoflurane.

EMG recordings. Nociceptive stimulation of different skin sites were performed using a $\mathrm{CO}_{2}$ laser (unfocused beam $3 \mathrm{~mm}$ in diameter, $600 \mathrm{~mJ}$; Irradia), once every 1.5-2 min. No visible damage of the skin or marked changes in response properties were detectable at these intensities. The needles used for EMG recordings were electrolytically pointed and insulated with a varnish coating except for $40 \mu \mathrm{m}$ at the tip. A small opening was made in the skin overlying the muscle, and the electrode was inserted into the midregion of each muscle belly. A reference electrode was inserted in an adjacent skin flap. Generally, the EMG activity in two or three hindlimb muscles was recorded simultaneously in each experiment. At least 25 consecutive receptive field mappings were performed for all the muscles in each animal.

Receptive field analysis. A topographical representation of receptive fields was used for illustrations (see Fig. $7 d$ ). For each muscle in each rat, responses on stimulation were normalized and expressed as percentage of the maximal response. From the measured data, $36 \times 76$ matrices were computed (intermediate sites were interpolated by spatial low-pass filtering). From these matrices, contour maps were constructed and plotted on the paw surface (using Surfer software from Golden Software). The resulting receptive fields were divided into three areas of sensitivity: maximal sensitivity $(70-100 \%$ of maximum), medium sensitivity $(30-70 \%$ of maximum), and low sensitivity ( $<30 \%$ of maximum) (Petersson et al., 2001).

Tail reflexes. C-fiber-mediated reflexes were elicited using a $\mathrm{CO}_{2}$ laser (pulse duration approximately two times reflex threshold). The reflexes were monitored with a high-speed camera (200 frames/s) (Petersson et al., 2003). The tail was stimulated laterally 10 times on each side of the distal $\sim 1 / 4$ of the tail. Reflexes were analyzed off-line on a PC using in-house software. The direction of reflexes in the horizontal plane and response latencies were determined. Tail-tip movements of $<0.02$ radians $(\sim 1 \mathrm{~mm})$ were not considered. Baseline skin temperature was $\sim 34^{\circ} \mathrm{C}$, as measured with a noncontact IR-radiation probe.

Reflex threshold measurements. The plantar side of the hindpaws was stimulated, a proximal and a distal site was tested on each side, and the display of a reflex response at any one of the four stimulated sites was judged as indicating the reflex threshold. The nociceptive thresholds were determined by using a $\mathrm{CO}_{2}$ laser with adjustable pulse duration (unfocused beam: diameter, $3 \mathrm{~mm}$; intensity, $15 \mathrm{~W}$; pulse duration, 8-50 $\mathrm{ms})$. The pulse duration was increased in steps of $2 \mathrm{~ms}$. The interstimulus interval was at least $30 \mathrm{~s}$. Tactile reflex thresholds were probed with calibrated Von Frey hairs up to $45 \mathrm{mN}$ (in animals older than P19, thresholds always exceeded $45 \mathrm{mN}$ ). The same stimulation sites were used for mechanical and thermal stimulation; the tactile testing always preceded the thermal testing.

Statistics and numerical transformations. Data from consecutive days were taken to represent matched observations. Friedman test was used to test whether the mean/median of any group was significantly different from the others. If a significant difference between means was found $(p<0.05)$, Dunn's multiple-comparison test was used to compare individual pairs of means. To directly compare digit 4 and heel data from B-HRP-injected animals of different ages, the dorsal-view maps from the different age groups were scaled to the P2 animals with respect to segmental and medial borders (rostrocaudally the length of the L1-L6 segments and mediolaterally the width of the dorsal horn). The scaling factors were as follows: $\mathrm{P} 8, \mathrm{RC}=1.58, \mathrm{ML}=1.85 ; \mathrm{P} 14, \mathrm{RC}=2.75, \mathrm{ML}=$ 2.19; $\mathrm{P} 21, \mathrm{RC}=2.94, \mathrm{ML}=2.50$; adult, $\mathrm{RC}=3.62, \mathrm{ML}=3.13$. The scaled dorsal-view maps were aligned after the caudalmost border of the heel projection. The images were transformed to binary bitmaps, and the coordinates of the focus of each termination field was calculated (defined as the center of gravity of the binary image) using software written in Matlab (MathWorks). The scaling process did not influence the digitization of data as confirmed by processing of images obtained from the same animal at $4 \times$ or $10 \times$ magnification, resulting in differences in estimated locations of termination centers of $\sim 1 \%$. We have previously shown that a simple and direct way of comparing the sensitivity distribution in receptive fields of two neurons or of two reflex pathways is to correlate the specific pattern of responses to stimulation of different predefined skin sites (Ekerot et al., 1991; Petersson et al., 2001). In the present study, correlations were calculated based on the averaged muscle responses evoked from 16 predefined skin sites in 10 consecutive mappings. The order of stimulations of these 16 skin sites was randomized between mappings. Because the sensitivity distributions of some muscles in certain animals did not pass a normality test (D'Agostino and Pearson omnibus normality test), nonparametric Spearman rank correlation was judged more appropriate than Pearson correlation. In the present study, all vehicle-treated animals had attained normal adult receptive fields (Holmberg and Schouenborg, 1996) when tested the fourth postnatal week. Therefore, the mean receptive field of this group was used as the reference (all individuals in the group had receptive fields highly correlated with this reference field).

\section{Results}

\section{Reorganization of afferent terminations}

To clarify to what extent the shape and relative location of termination fields of afferents from different skin sites are established already at birth, the termination patterns of myelinated fibers in the horizontal plane in laminas III-IV (where cutaneous tactile afferents are known to terminate) were mapped during development in 56 rats of different age by subcutaneous injections of B-HRP in the plantar hindpaw (for controls on the specificity of the tracer and number of dorsal root ganglion neurons labeled at different ages, see supplemental material, available at www. jneurosci.org). The study focused on injections made into the heel $(n=37)$ and distal part of digit $4(n=33)$. Additional sites, such as digit $2(n=4)$ and the proximomedial pad $(n=4)$, were initially also injected to ascertain that the sites chosen were representative. On the gross scale, skin afferents from the sites studied terminated approximately in the same segmental areas in the younger pups as they did in the adults. However, several clear differences between juvenile and adult animals were observed. The termination fields were larger and displayed a much greater interindividual variance in termination patterns in the younger pups than in adults (Fig. 1a). From P8 to P14, the termination patterns gradually changed in shape and became more adult-like, and at P14, the pups exhibited a near-adult pattern of labeling. A detailed analysis revealed that, for example, the location of the focus of the termination area (defined as the "center of gravity") of digit 4 in relation to that of the heel varied by up to $\sim 0.5 \mathrm{~mm}$ along the rostrocaudal axis in the newborn animals, corresponding to approximately half a segment. Noticeable differences in location of termination field foci along the mediolateral axis were also evident. This interindividual variation became less evident with age (Fig. $1 b$ ). Control experiments were done in 11 rats [P2 $(n=2), \mathrm{P} 14(n=5)$, and adult $(n=4)]$ to assess how much of this variance that could be attributable to factors like inadvertent variations in the precise location of injection sites or peripheral tracer spreading. Interestingly, even for the youngest pups, bilateral staining of corresponding sites of the hindpaws resulted in almost mirror-image labeling patterns in the two hemicords (Fig. 2). Only minor differences with regard to, e.g., mediolateral extent of termination fields could be found when examining rightleft asymmetries. Control experiments in which the plantar nerves were cut were also made to further assess the spreading of tracer substance in the periphery. In these latter animals, no terminals in the dorsal horn were labeled after injections in the central pads $(n=2)$ and midplanta $(n=3)$. Hence, the large differences in termination patterns found between individuals of 
a

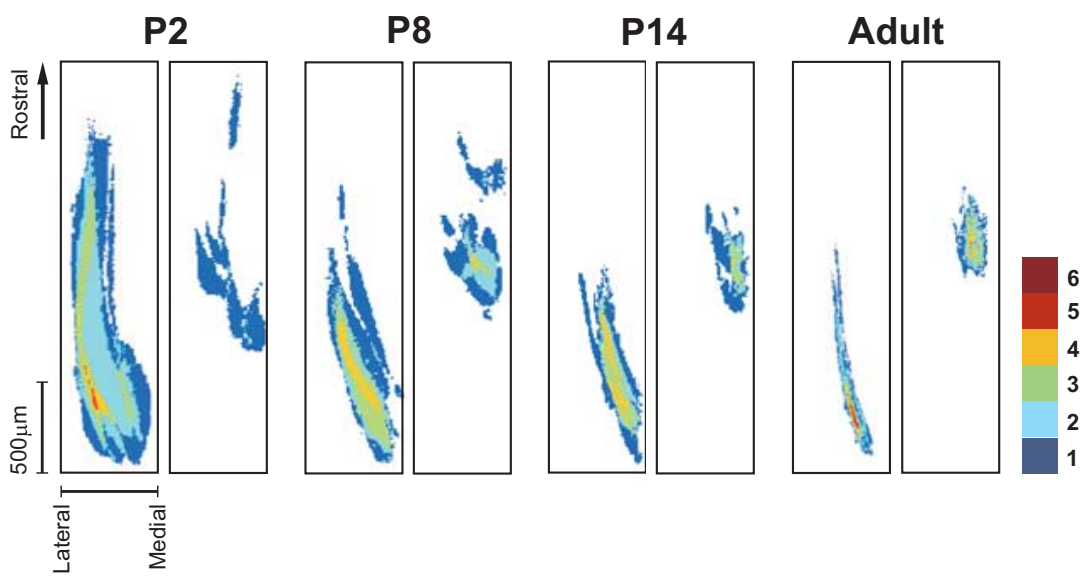

b

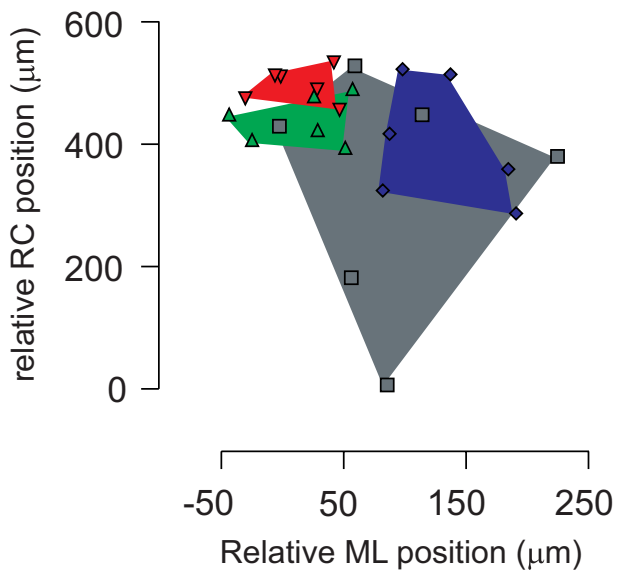

Figure 1. Large and highly variable primary afferent termination fields in neonate rats are reorganized during development. $\boldsymbol{a}$, Superimposed labeling patterns from six animals in each age group after subcutaneous tracer injections in the left heel (left) and in the right digit 4 (right). Labeled termination areas in each animal were rostrocaudally aligned with respect to the caudalmost border of the heel projection. The right border in each panel indicates the medial border of the dorsal horn (mediolateral orientation is reversed in right panels to mimic left-side labeling patterns). The color code in each pixel denotes the number of animals in which labeled terminals were found in the corresponding area. $\boldsymbol{b}$, Relative location of termination foci. RC and ML locations of digit 4 termination foci relative to that of the heel in individual animals for different postnatal ages are shown. Positive RC and ML values indicate that the digit 4 focus is located rostral and medial to that of the heel focus, respectively. All ages are scaled to P2 spinal cord size (for scale factors and calculation of coordinates of foci, see Materials and Methods).
P2

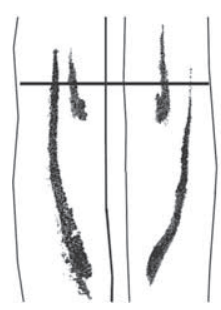

P14

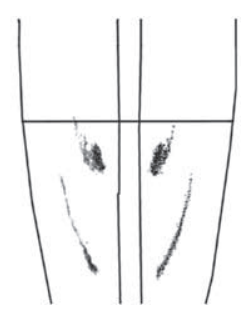

Adult

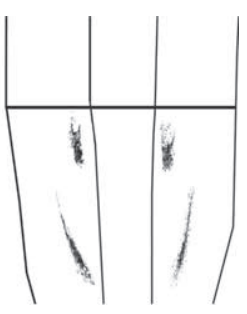

Figure 2. Symmetrical bilateral labeling patterns in individual animals. Samples of dorsal-view maps of termination patterns in left and right dorsal horn laminas III-IV after bilateral injections of B-HRP into the heel and digit 4 in single P2, P14, and adult animals. All images are scaled to P2 spinal cord size and rostrocaudally aligned with respect to the caudal heel termination field.

the same neonatal age cannot, to any significant extent, be explained by variations in the precise location of different skin injections, nor by differences in spread of tracer in the skin. Moreover, other control experiments showed that a similar number of dorsal root ganglion cells were stained in the different age groups, indicating that the large differences in labeling patterns between age groups cannot be explained by, for example, reduced tracer uptake and axonal transport with age (supplemental Fig. 2 , available at www.jneurosci.org as supplemental material). This notion is also supported by the finding described below that pharmacological blocking of NMDA channels in the lumbar spinal cord during P6-P21 appear to preserve the neonatal termination pattern.

The gradual shrinkage of termination fields during the first two postnatal weeks found is thus indicative of a process of pruning of inaccurate axonal arbors. What is more, the fact that the variation in relative termination fields of different hindpaw sites decreases considerably with age indicates that the termination field can move a considerable distance during development. This suggests that new axon collaterals may also be established. To examine this latter possibility further, the same skin site was injected twice with $\mathrm{CTb}$ conjugated to two different fluorophores with a $4 \mathrm{~d}$ interval (P1/P5, P7/P11, and P13/P17) and evaluated at $\mathrm{P} 8, \mathrm{P} 14$, and $\mathrm{P} 21$, respectively (Fig. $3)$. In the $\mathrm{P} 21$ group, injections in digit 4 gave rise to a very specific area of labeling with almost complete overlap of labeling from the two injections; i.e., the same terminals were labeled twice. In contrast, labeling overlapped much less in the younger animals, suggesting substantial ingrowth of new terminal arbors during the first 2 weeks (Fig. 3, green tracer). In addition, for some terminal regions, the axons labeled by the first tracer (Fig. 3, red tracer) did not transport the second tracer. This latter finding may suggest that transport mechanisms are halted some time before the pruning of inadequate axon branches occurs. Control experiments were done to exclude the possibility that the two tracers interacted in the neonate, e.g., that one tracer blocked the uptake of the other (supplemental Fig. 3, available at www.jneurosci.org as supplemental material).

Together, these experiments indicate that the early postnatal developmental phase is characterized by a floating body representation with regard to coarse fiber input with substantial reorganization through gradual establishment and elimination of axonal arbors up to the third week. This conclusion is further strengthened by the demonstration that the changes in termination fields found are activity dependent (see below).

\section{Alignment of thin and coarse fiber terminations}

In adults, sensorimotor circuits usually receive a congruent multisensory input (Levinsson et al., 2002). Considering that thin and coarse nerve fibers grow into the dorsal horn at different time points and by different routes (Jackman and Fitzgerald, 2000) and the lack of a clear somatotopic organization in the dorsal root ganglia (Prats-Galino et al., 1999), it is puzzling how the different 


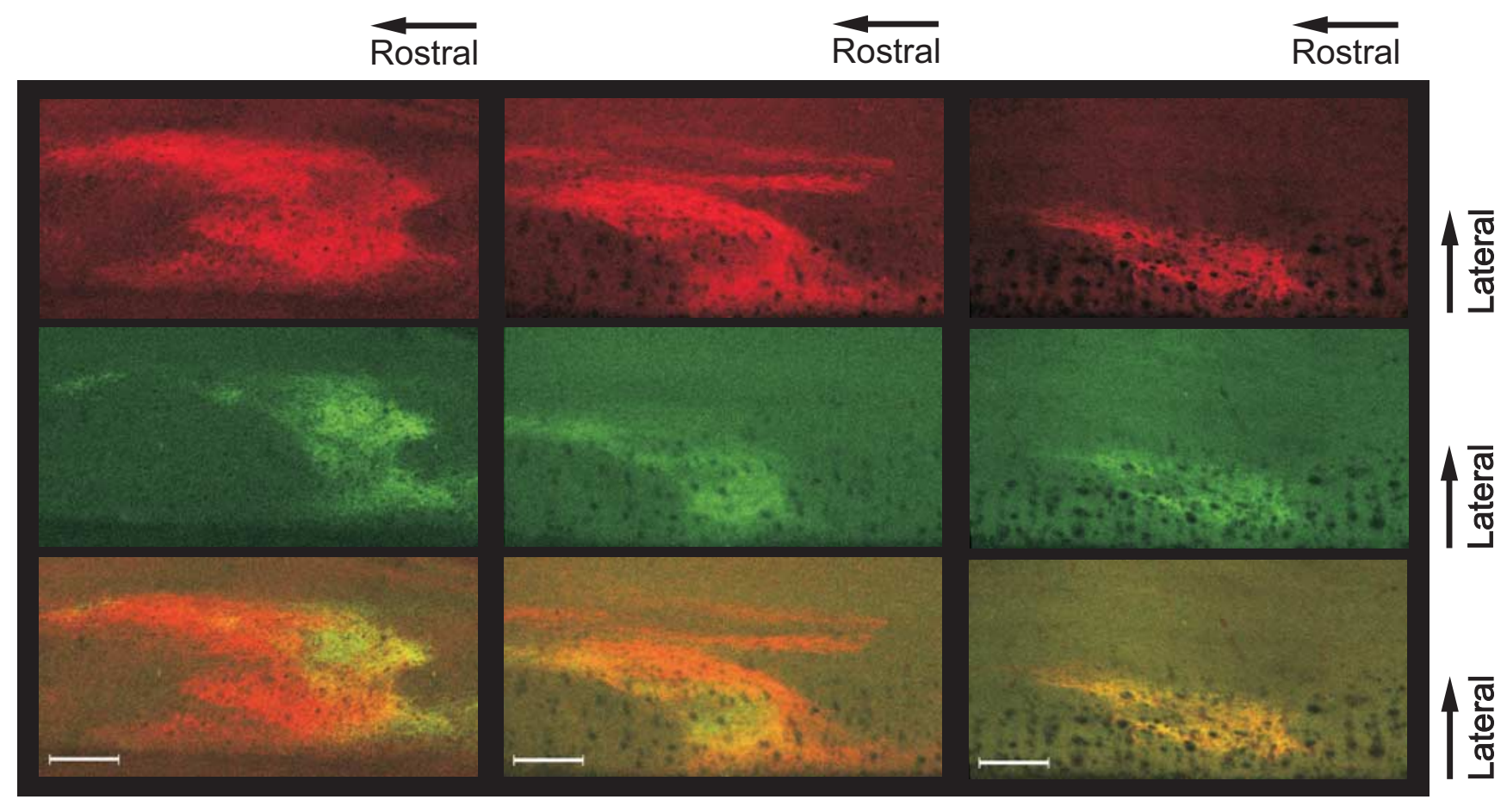

\section{P1-P8}

P8-P14

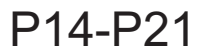

Figure 3. Changes in individual terminal field morphology during the first 2 weeks. Three animals of different age received consecutive digit 4 labeling at different time points. Left, A P8 animal injected with red CTb at P1 and green CTb at P5. Middle, A P14 animal injected at P8/P11. Right, A P21 animal injected at P14/P18. Note the large nonoverlapping areas in the P8 and to some extent the P14 animal, but almost complete overlap at P21. Scale bars, $100 \mu \mathrm{m}$.

types of fibers from a given body part are able to locate and make functional connections to the adequate cells in the dorsal horn during development. It was therefore of interest to study the process whereby the thin and coarse primary afferent terminations are aligned in the dorsal horn. Both thin and coarse primary afferent fibers from the receptive field focus of the nociceptive withdrawal reflex (NWR) of the peroneus longus muscle (PL) were labeled (Fig. 4a). The termination patterns of afferent terminals were mapped at different postnatal ages (P3-P24) in 50 rat pups using WGA and $\mathrm{CTb}$ (for control experiments on tracer specificity, see supplemental Fig. 1, available at www.jneurosci.org as supplemental material). In the neonates, there was an extensive laminar overlap in large regions of the dorsal horn. In the overlap zone, terminals from thin and coarse fibers were found in close proximity and densely packed together in the space between cell bodies. A gradual dorsoventral separation with $\sim 6.2 \mu \mathrm{m} / \mathrm{d}$ (from an initial mean overlap of $144 \mu \mathrm{m}$ at P3) followed during the subsequent weeks, and by the third or fourth week, the coarse fibers terminated almost exclusively directly ventral to the thin fibers (Fig. $4 b$ ). At the same time, the termination fields were reorganized mediolaterally from a broad unorganized pattern into a thin quite-conspicuous columnar structure (mediolateral width of the overlap zone decreased with $\sim 6.5$ $\mu \mathrm{m} / \mathrm{d}$ from an initial mean width at P3 of $163 \mu \mathrm{m}$ ) (Fig. $4 c$ ). Changes occurred in parallel for thin and coarse fibers. In the newborn animals, the coarse fibers formed a very characteristic circle-shaped structure in the dorsoventral-mediolateral plane. By collecting images at different time points after the peripheral injection of tracer, it was shown that the circle consisted of both a lateral and a medial projection joining up in the dorsal horn (Fig. 5). Interestingly, the two projections merged in a region just ven- tral to the region later most heavily innervated by thin fibers from the same skin area (this pattern was found also for other parts of the plantar paw, such as the distal parts of the digits or centrally in the planta; $n=5$ ). By the third week, only the lateral termination field remained (this was studied in detail in a subgroup of animals exhibiting very strong CTb labeling; medial terminations were detected: week 1 in 12/12 rats, week 2 in 6/6 rats, and week 3 in $2 / 8$ rats). The thin fibers also terminated in a widely distributed manner together with coarse fibers in the newborn but were later reorganized in conjunction with the coarse fibers (mean mediolateral width: week 1, $510 \mu \mathrm{m}$; week 2, $500 \mu \mathrm{m}$; week 3, $241 \mu \mathrm{m}$; week 4, $143 \mu \mathrm{m}$ ) (Fig. 4d). At the end of the third week, the WGA-labeled terminals of lamina II and the CTb-labeled terminals of lamina III tended to align in an even finer microcolumnar structure within the larger column, in line with previous suggestions of a higher level of organization of different sensorimotor circuits (Scheibel and Scheibel, 1968; Rethelyi and Capowski, 1977; Levinsson et al., 2002).

\section{Reorganization is activity dependent}

Because the functional fine tuning of the sensorimotor transformation in the reflex circuits is activity dependent (Holmberg et al., 1997; Petersson et al., 2003; Waldenstrom et al., 2003), we wanted to clarify whether this applies also to the postnatal reorganization of termination patterns. Because NMDA receptors play a critical role in experience-dependent developmental plasticity (Huang and Pallas, 2001; Luthi et al., 2001; Lee et al., 2005) and chronic application of the NMDA receptor antagonist MK801 is known to affect the laminar termination of coarse fibers (Beggs et al., 2002), we first evaluated the effect of MK-801 applied topically to the lumbar spinal cord P6-P21 using Elvax 


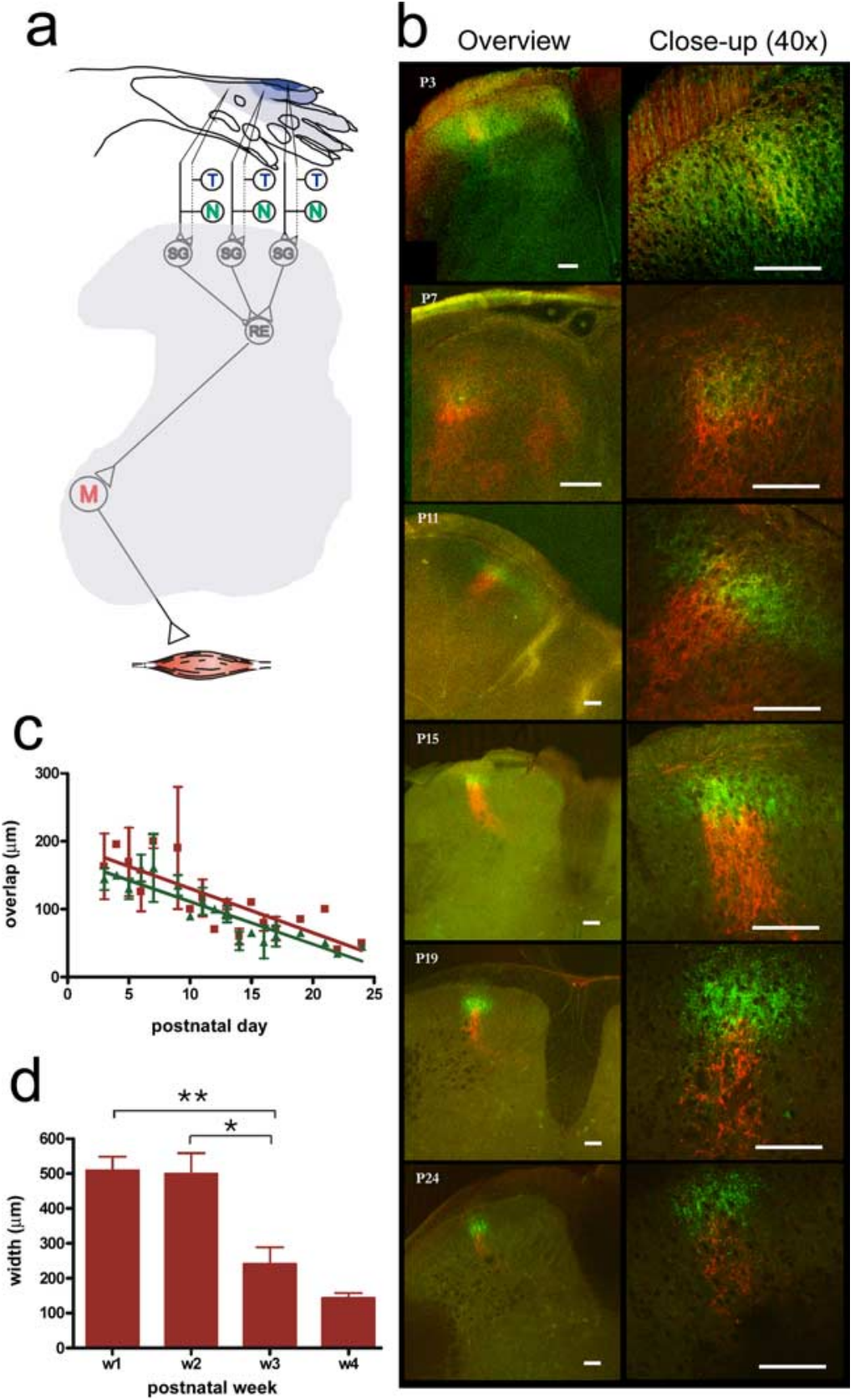

Figure 4. Developmental reorganization of coarse and thin afferent terminations from the NWR receptive field of the PL. $\boldsymbol{a}$, Schematic of the immature PL NWR module: note the tactile-nociceptive convergence in the superficial dorsal horn. T/N, Tactile/nociceptive; SG, substantia gelatinosa interneurons; RE, reflex encoding multimodal interneurons; $M$, motor neurons. The quantified PL NWR receptive field (top) is shown as blue shaded isoresponse areas; colors denote $>70 \%, 30-70 \%$, and $0-30 \%$ of maximum values. $\boldsymbol{b}$, Examples of images collected at different developmental time points (P3-P24). CTb-labeled fibers (red) traverse the WGA (green)-labeled zone in the first 2 weeks. During the third week, the laminar overlap decreases, and terminal fields narrow mediolaterally into a columnar structure. c, The mediolateral (red) and dorsoventral (green) maximum width of the overlap zone of thin and coarse afferents (postnatal days 3-24; linear regression slopes $/ 0 ; p<0.0001$ ). $\boldsymbol{d}$, Mediolateral maximum width of the thin fiber terminal field during postnatal development ( $p<0.0001$, Kruskal-Wallis test, ${ }^{*} p<$ $0.05 ;{ }^{* *} p<0.01$, Dunn's multiple-comparison post test. Scale bars, $100 \mu \mathrm{m}$.

implants. These experiments showed that MK-801-treated animals $(n=15)$ exhibited much more extensive labeling patterns along the rostrocaudal axis [1410 $\pm 440 \mu \mathrm{m}$ (mean \pm SD) compared with $380 \pm 70 \mu \mathrm{m}$ for vehicle-treated animals $(n=5)]$ and typically also along the mediolateral axis $(320 \pm 70 \mu \mathrm{m}$ compared with $180 \pm 50 \mu \mathrm{m})$ at $\mathrm{P} 21$, strongly resembling the neonatal terminal fields (Fig. 6). Furthermore, the locations of the termi- nal foci varied much more in the MK-801-treated animals (interindividual differences in mediolateral position of up to $\sim 350 \mu \mathrm{m}$ compared with $\sim 60 \mu \mathrm{m}$ in vehicle-treated rats).

At the reflex level, lumbar administration of MK801 distorted the sensitivity distribution of NWR receptive fields of the plantar paw, whereas all vehicletreated animals developed normal adult receptive fields (Holmberg and Schouenborg, 1996) (Fig. 7d). Similarities of receptive fields to the normal adult pattern, as assessed by Spearman rank correlations between receptive fields of individual MK-801treated animals and the mean receptive fields of the vehicle group (see Materials and Methods for details), were as follows for PL/extensor digitorum longus (EDL): $0.45 \pm 0.17 /-0.21 \pm 0.22$ compared with $0.95 \pm 0.03 / 0.79 \pm 0.08$ for vehicle-treated animals [mean $\pm \mathrm{SD}$; number of receptive fields: (PL/EDL) vehicle, 4/4; MK-801, 6/6; each field was calculated as the average of 10 consecutive receptive field mappings]. Similarly, chronic MK-801 treatment of the sacral cord by Elvax implantation at P5 increased the error rate of tail NWRs at the end of both the second and third week (Fig. 7b). In contrast, after lumbar implantation, both MK-801-treated $(n=15)$ and vehicle-treated $(n=8)$ animals showed normal functionally adapted tail withdrawal reflexes at P21 (mean \pm SD error rates: $1 \pm 2 \%$ and $2 \pm 4 \% \mathrm{MK}-801$ and vehicle, respectively), indicating that MK-801 acts primarily on the segments underneath the implant. These data show that both the primary afferent termination fields and the receptive fields of NWRs are functionally adapted via NMDA-dependent mechanisms. Interestingly, we found no difference in reflex thresholds after MK-801, indicating a preferential effect on the mechanisms underlying the spatial organization of the terminations and somatosensory imprinting of NWR receptive fields.

Because tactile feedback on spontaneous movements has been shown to be instrumental for fine tuning of receptive fields in NWR modules, we then tested the effect of adding on constant tactile noise over large skin areas by placing pups together with their mother in a vibrating cage for increasing time periods from P3 and on (vibration frequency $\sim 200$ $\mathrm{Hz}$,) (Gescheider et al., 2004). The tactile input, unrelated to spontaneous movements, present in this situation was hypothesized to disturb adequate receptive field formation, leading to smeared receptive fields and inappropriate reflex responses. Six vibrated litters were compared with six age matched control litters with respect to primary afferent termination fields and NWR receptive field characteristics. In vibrated animals $(n=35)$, a marked terminal overlap in the superficial dorsal horn of thin and coarse fibers remained during the third and fourth weeks (P13-P24 was examined; mean mediolateral/dorsoventral maximum width: week 3, 154/136 $\mu \mathrm{m}$; week 4, 73/82 $\mu \mathrm{m}$; compared with controls: week 3, 78/56 $\mu \mathrm{m}$; week $4,47 / 42 \mu \mathrm{m})$ and was even more evident in the more caudal segments (Fig. 8). A weak scatter of CTb-labeled terminals in the medial part of the deeper dorsal horn also remained in most animals (week 3 in 9/13 rats and week 4 in 2/3 rats), reminiscent of the double projection 
normally present during the first postnatal week (see above). The thin fiber terminations were also markedly affected by vibration and generally colocalized with the coarse fiber terminations in broadened terminal fields $(p<0.001$, Mann-Whitney for weeks 3 and 4; mediolateral width: week 3, $309 \pm 58 \mu \mathrm{m}$ compared with $241 \pm 48$ $\mu \mathrm{m}$; week 4, $218 \pm 55 \mu \mathrm{m}$ compared with $143 \pm 15 \mu \mathrm{m}$ in vibrated and controls, respectively).

The expanded termination fields of both coarse and thin fibers could possibly reflect unspecific afferent connections to multiple sensorimotor circuits as a result of the synchronous input fed to large skin areas. This notion is further supported by the finding that the vibrated rats $(n=7)$ exhibited NWR receptive fields with relatively strong excitatory input from skin regions normally inhibiting muscle activation (Fig. $7 d)$. In these peripheral parts of the receptive fields, vibrated animals also displayed a high interindividual variability (encircled in Fig. 7c). Finally, the tactile reflex threshold was strongly increased in vibrated animals compared with controls $[p<0.001$, Friedman test and Dunn's multiplecomparison test; median values (P8-P19), 41 and $12.8 \mathrm{mN}$ for vibrated and controls, respectively]. In contrast, no significant effect on nociceptive reflex thresholds was found ( $p>0.05$, Friedman test; median values (P8-P19), 17.8 and $20.4 \mathrm{~ms}$ for vibrated and controls, respectively). This may suggest the presence of modalityspecific developmental regulatory mechanisms of neuronal connections strengths. This latter assumption was also supported by experiments involving a group of animals ( $n=20$ from five litters) that received capsaicin treatment at birth $(50 \mathrm{mg} / \mathrm{kg}$; abolishing most of the thin fiber input), selectively increasing their nociceptive $[p<$ 0.001 , Friedman test and Dunn's multiplecomparison test; median nociceptive threshold (P8-P19), $26.2 \mathrm{~ms}$ ] but not tactile withdrawal reflex thresholds $[p>0.05$, Friedman test; median tactile threshold (P8-P19), $22.3 \mathrm{mN}$ ].

\section{Discussion}

The data presented indicate, contrary to previous assumptions (Smith, 1983; Fitzgerald, 1987; Mirnics and Koerber, 1995b; Silos-Santiago et al., 1995), that the body representation in the neonate rat is far from adult-like and that a period of extensive remodeling of primary afferent connections follows during the first 3 postnatal weeks. Accordingly, as primary afferents first grow into the spinal cord, a crude and floating somatotopic map characterized by considerable overlap, large variations between animals, and numerous erroneous connections is established. Nevertheless,

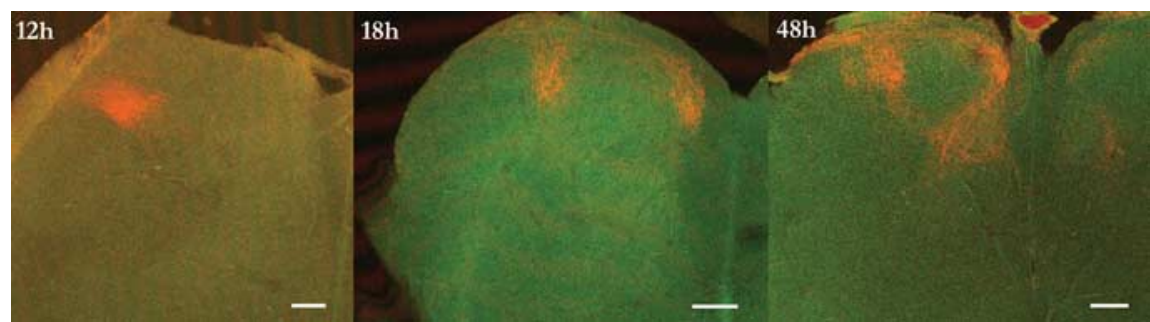

Figure 5. Termination fields of coarse fibers in the neonate. Transverse sections were taken 12, 18, and $48 \mathrm{~h}$ after skin CTb injection. Two parallel projections were visible after $18 \mathrm{~h}$. Scale bars, $100 \mu \mathrm{m}$.

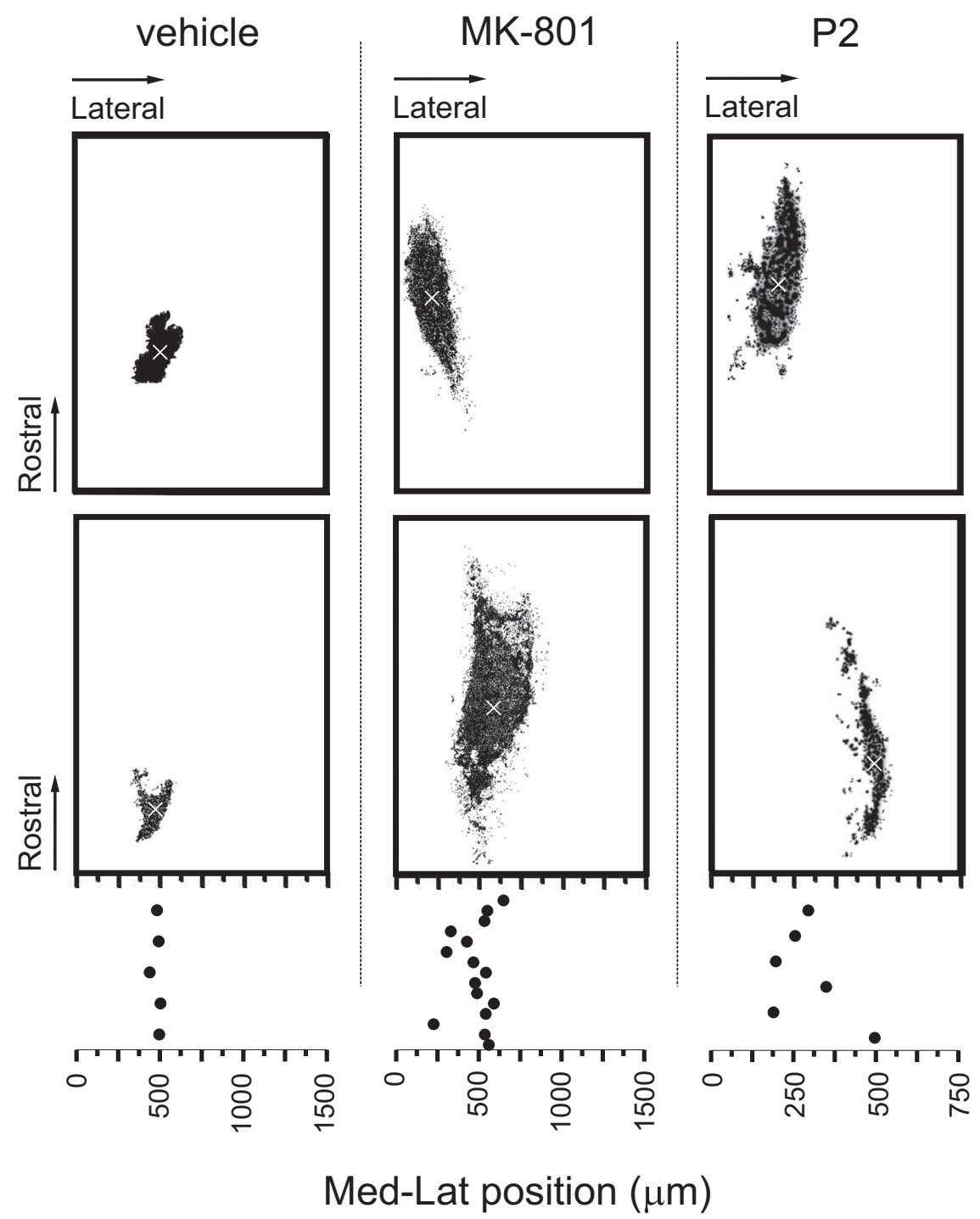

Figure 6. Pharmacological manipulations disrupt the refinement of terminal fields. Samples of digit 4 terminations in P21 animals treated with either vehicle (left) or MK-801 (middle) and P2 animals (right; scaled to P21 size) as a reference. Top and bottom panels in each treatment group show the results obtained from two different animals. Mediolateral (Med-Lat) positions from midline of the terminal foci ( $x$-marked) in individual animals are plotted below. Note the large interindividual variation in MK-801-treated animals and in P2 pups.

mirror-image termination patterns for corresponding skin sites were found in the right and left spinal hemicords in individual neonates, indicating that, for given conditions, the ingrowth of the primary afferents is to a large extent determined by genetic programs. Assuming that competition for space and trophic support are factors of critical importance for the establishment and 


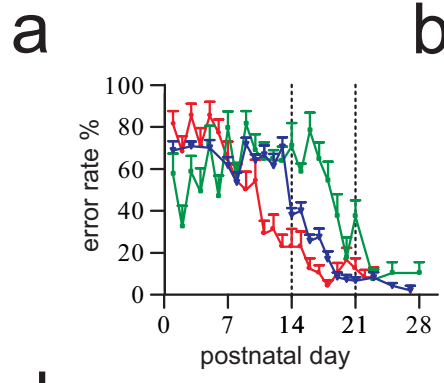

b
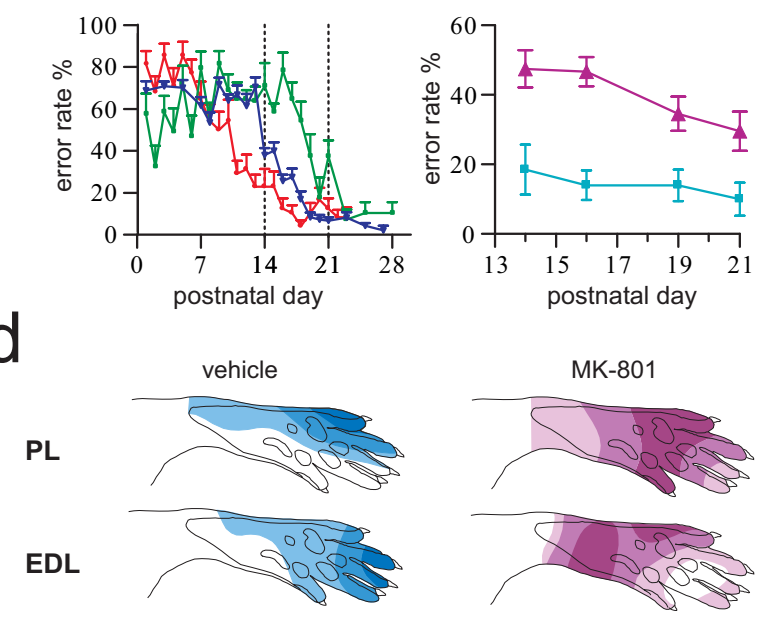

Figure 7. Pharmacological and sensory manipulations disrupt functional adaptation of sensory processing in the nociceptive withdrawal reflex. $\boldsymbol{a}$, Normal developmental functional adaptation of tail withdrawal reflexes in the horizontal plane in three litters. Error rate denotes the proportion of erroneous reflex movements, i.e., toward instead of away from the nociceptive stimulus [modified from Waldenstrom et al. (2003)]. $\boldsymbol{b}$, MK-801-treated animals $(\boldsymbol{\Delta} ; n=12$ ) displayed significantly higher tail reflex error rates than vehicle-treated pups $(\square ; n=10 ; p=0.029$; Mann-Whitney). $\boldsymbol{c}$, The mean number of action potentials and the corresponding SD for 16 standard sites are plotted for PL [vibrated (gray; $n=7$ ), vehicle (cyan; $n=4)$, and MK-801 (purple; $n=6$ )]. The average spontaneous activity recorded before stimulation has been subtracted from poststimulus values; as a consequence, inhibition results in a negative number of action potentials. It can be seen that the vibration-treated pups displayed large interindividual response variability close to the receptive field periphery (encircled values). Note also the expansion of the excitatory receptive fields into skin areas normally producing inhibition, in vibrated and MK-801-treated animals. $\boldsymbol{d}$, Examples of quantified NWR receptive fields of PL and EDL obtained during the fourth postnatal week. MK-801-treated and vibrated animals exhibited inappropriate and flattened receptive fields, respectively. Vehicle-treated animals displayed normal adult-like receptive fields [in agreement with previous results (Holmberg and Schouenborg, 1996)]. Illustrated receptive fields were constructed from quantified EMG responses (mean of 10 mappings) by stimulation of 16 standardized skin sites; values from intermediate sites were interpolated by spatial low-pass filtering.

preservation of synaptic connections (Fitzgerald, 2005), it is conceivable that the initial variation between animals is attributable to factors such as innervation density (Brown et al., 2004), dorsal root ganglion cell death (Oppenheim, 1991), reorganization of dorsal root ganglion cells (Knaut et al., 2005), and relative timing of primary afferent ingrowth from different skin areas (Jackman and Fitzgerald, 2000). Our data, in particular, indicate an important role for central plastic mechanisms, and although minor changes in peripheral projections could also contribute to the floating body representation in young animals, previous studies have shown that the nerves innervating the skin areas studied are in place already at birth (Mirnics and Koerber, 1995a; Jackman and Fitzgerald, 2000).

The process of afferent reorganization was found to include both elimination of erroneous connections and establishment of new connections, and newly ingrown terminations in young animals often did not overlap with previous labeling (see also Ruthazer et al., 2003). Because both the functional adaptation of the NWR receptive fields and the reorganization of the cutaneous terminations were found to depend on NMDA receptor mechanisms and tactile input and occurred during the same time period, the postnatal reorganization of the NWRs may reflect, at least in part, structural changes induced by activity-dependent mechanisms. Similar changes in axonal termination patterns, being a part of learning-dependent processes during development, have been found in, e.g., the sound localization system in barn owls (Linkenhoker et al., 2005). It may also be worth noting that MK-801 treatment did not alter reflex thresholds despite the dis-
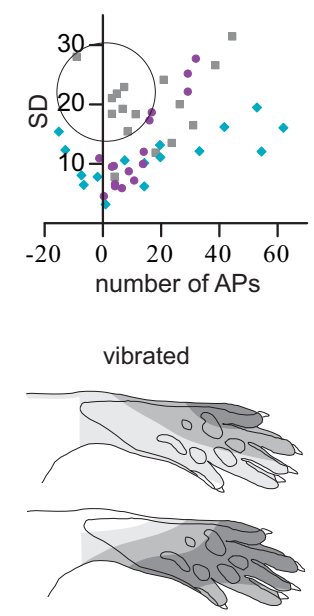

tortion of the NWR receptive fields and afferent terminations. Hence, the mechanisms underlying somatosensory imprinting and the mechanisms controlling the overall sensitivity appear to be different.

Because the adult somatotopic organization in the lower lumbar cord is related to the receptive fields of withdrawal reflex circuits (Levinsson et al., 2002), somatosensory imprinting induced by spontaneous movements (Petersson et al., 2003) may play a critical role in the postnatal rewiring. This mechanism relies on cross-modality learning and may thus require a multisensory alignment (Levinsson et al., 2002; Petersson et al., 2003; Waldenstrom et al., 2003). For this reason, it was of interest to analyze how the alignment of the multisensory input is established. From the present study, it appears that myelinated and unmyelinated cutaneous fibers from the same skin site contact neurons in the same area or possibly even the same postsynaptic cells in the superficial dorsal horn during early postnatal life. At that age, termination fields of the two fiber groups are large and unorganized. However, they occupy approximately the same area in the spinal cord, indicating an early grouping mechanism for afferents from the same part of the body. Whereas our data support previous observations that the dorsal root ganglia are not somatotopically organized, coarse and thin axons from the same skin site appear to run in bundles in the peripheral nerves and also in the root entry zone. One could thus speculate that afferent fibers of different size from the same part of the body or located within the same nerve fascicle exhibit similar sensitivity to gradients of trophic substances or regulatory factors, which would guide central terminations, somewhat resembling the assembly of motor neuron columns (Jessell, 2000; Ladle et al., 2007). Another interesting possibility is that low-threshold myelinated cutaneous fibers, which enter the spinal cord first (Altman and Bayer, 1984), could have a guiding role for other cutaneous afferents (Petersson et al., 2003; Waldenstrom et al., 2003). This notion is supported by the present finding that an altered tactile termination induced by vibration was accompanied by a corresponding change of the thin fiber termination. It may be of relevance in this context that coarse fibers from the plantar skin display a characteristic circular structure in transverse sections of the lower lumbar cord the first two postnatal weeks. Time-lapse experiments showed that this pattern arises as two major parallel projections merge in the dorsal horn. This union of coarse afferents from the same part of the body, with similar conduction velocities, potentially enables coincidence detection or spike timing-dependent plasticity. If thin fibers enter the spinal cord together with myelinated $(\mathrm{A} \beta / \mathrm{A} \delta)$ fibers from approximately the same part of the body through the lateral entry zone, a refinement via experience-dependent selection of connections in the dorsal horn receiving synchronized input via the medial and lateral pathways could follow. In addition, the proximity of fibers from identical skin areas may allow cross talk between coarse and thin fibers during early develop- 
a

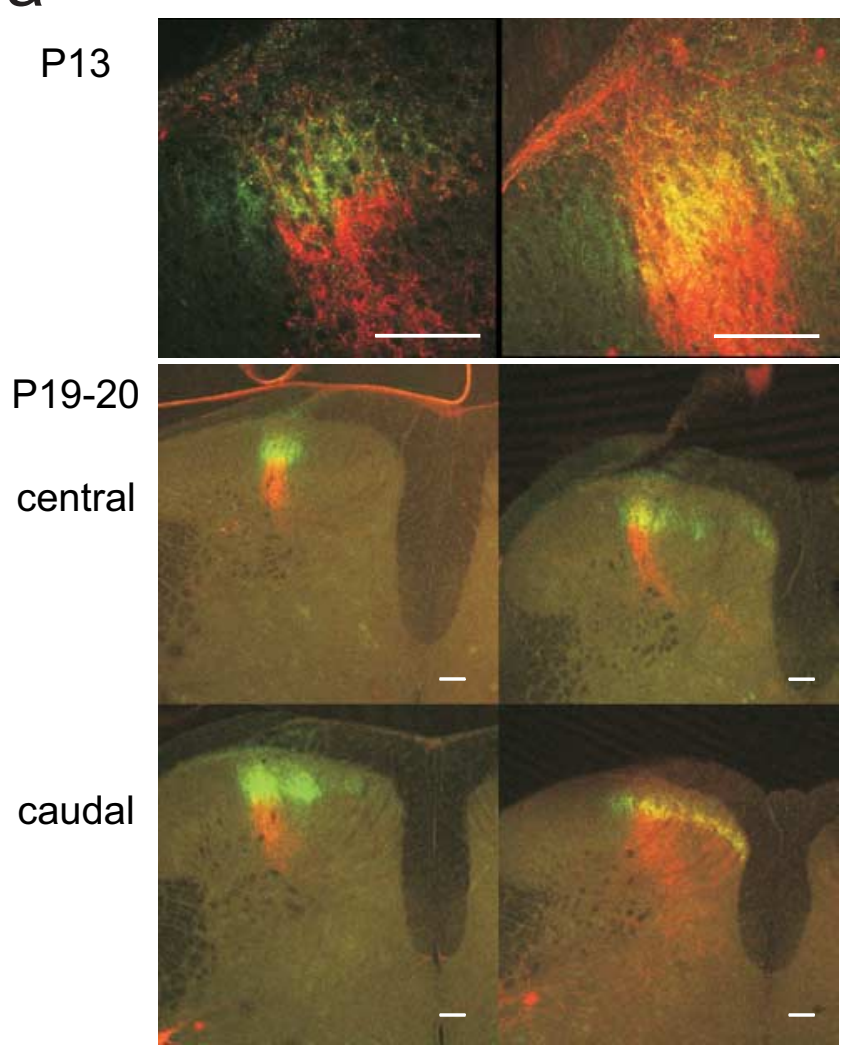

b

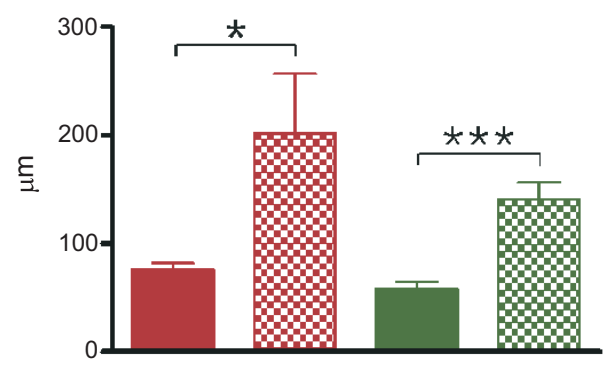

$\mathrm{ML}$

Figure 8. Sensory manipulations disrupt refinement of terminal fields. $\boldsymbol{a}$, In vibrated rats, the large overlap between thin and coarse fibers, normally present only in the neonate, remained (samples from end of second and third weeks shown). Overlap was often strongest in the caudal segments. A weak medial scatter of CTb-labeled terminals also persisted during the first 3 weeks in the majority of vibrated animals. Red, CTb; green, WGA. Scale bars, $100 \mu \mathrm{m} . \boldsymbol{b}$ Terminal overlap of thin and coarse fibers is significantly larger in rats vibrated $(V)$ from birth than in age matched (P13-P24) controls (C) mediolaterally (ML; red) and dorsoventrally (DV; green; ${ }^{*} p<0.05 ;{ }^{* *} p<0.001$, Mann-Whitney).

ment (Amir et al., 2005) that potentially also would favor multisensory convergence onto the same spinal neurons through Hebbian-type plasticity (Katz and Shatz, 1996; Meyer and Smith, 2006). Our present finding that the alignment process is, at least partly, NMDA dependent could be indicative of such cellular mechanisms.

One of the last steps in the establishment of the adult body representation is a segregation of afferents of different modalities into different laminas (Park et al., 1999; Nakatsuka et al., 2000; Fitzgerald, 2005). The present study demonstrates that this phase overlaps in time with the "somatotopic" reorganization described above but appears to end some days later. Moreover,

although the type of target cells for different types of afferent fibers may be partly under genetic control (A. I. Chen et al., 2006; C. L. Chen et al., 2006), the present data indicate that activitydependent mechanisms are also important, because continuous tactile stimulation (vibration treatment) maintained the laminar overlap and affected both the topographical reorganization and reflex adaptation up to the fourth postnatal week. This conclusion is also supported by the finding that spinal MK-801 treatment interferes with laminar separation (Beggs et al., 2002). It is known that increased neuronal activity leads to a rapid increase in the expression of NGF and BDNF mRNA, the natural ligands to the TrkA and TrkB receptors responsible for survival and phenotypic development of subgroups of cutaneous afferents, as well as altered expression of Trk receptors in the plasma membrane (Poo, 2001). The expression of Trk receptors normally changes during postnatal development (Guo et al., 2001) and could thus be important for the diminished overlap of thin and coarse fibers in the superficial dorsal horn during the third week. In addition, they may also play a role in activity-dependent synaptogenesis that helps to guide axon arbor growth during development (Meyer and Smith, 2006). It is therefore tempting to speculate that an abnormal expression of Trk receptors and neurotrophins after unspecific tactile input or activity blockade could contribute to the disturbed body representation seen in the present study.

The profound structural reorganization of the multisensory afferent termination in the dorsal horn during postnatal development occurs in parallel with the functional adaptation in sensorimotor circuits and may thus, at least in part, reflect the establishment of an action-based body representation already at the first synaptic relay. An action-based body representation greatly simplifies and speeds up the complex task of transforming intricate multimodal sensory patterns into appropriate corrections of movements. The present demonstration of an action-based sensory encoding arising from a transitory floating somatotopy may thus also apply to other sensorimotor systems and is likely to have implications in other fields in which sensory input needs to be integrated with motor control, such as in the emerging field of neurorobotics.

\section{References}

Altman J, Bayer SA (1984) The development of the rat spinal cord. Adv Anat Embryol Cell Biol 85:1-164.

Amir R, Kocsis JD, Devor M (2005) Multiple interacting sites of ectopic spike electrogenesis in primary sensory neurons. J Neurosci 25:2576-2585.

Beggs S, Torsney C, Drew LJ, Fitzgerald M (2002) The postnatal reorganization of primary afferent input and dorsal horn cell receptive fields in the rat spinal cord is an activity-dependent process. Eur J Neurosci 16:1249-1258.

Brown PB, Koerber HR, Millecchia R (1997) Assembly of the dorsal horn somatotopic map. Somatosens Mot Res 14:93-106.

Brown PB, Koerber HR, Millecchia R (2004) From innervation density to tactile acuity: 1. Spatial representation. Brain Res 1011:14-32.

Chen AI, de Nooij JC, Jessell TM (2006) Graded activity of transcription factor Runx3 specifies the laminar termination pattern of sensory axons in the developing spinal cord. Neuron 49:395-408.

Chen CL, Broom DC, Liu Y, de Nooij JC, Li Z, Cen C, Samad OA, Jessell TM, Woolf CJ, Ma Q (2006) Runxl determines nociceptive sensory neuron phenotype and is required for thermal and neuropathic pain. Neuron 49:365-377.

Coggeshall RE, Jennings EA, Fitzgerald M (1996) Evidence that large myelinated primary afferent fibers make synaptic contacts in lamina II of neonatal rats. Brain Res Dev Brain Res 92:81-90.

Dederen PJ, Gribnau AA, Curfs MH (1994) Retrograde neuronal tracing with cholera toxin B subunit: comparison of three different visualization methods. Histochem J 26:856-862. 
Ekerot CF, Garwicz M, Schouenborg J (1991) Topography and nociceptive receptive fields of climbing fibres projecting to the cerebellar anterior lobe in the cat. J Physiol (Lond) 441:257-274.

Fitzgerald M (1987) Prenatal growth of fine-diameter primary afferents into the rat spinal cord: a transganglionic tracer study. J Comp Neurol 261:98-104.

Fitzgerald M (2005) The development of nociceptive circuits. Nat Rev Neurosci 6:507-520.

Gescheider GA, Bolanowski SJ, Verrillo RT (2004) Some characteristics of tactile channels. Behav Brain Res 148:35-40.

Guo A, Simone DA, Stone LS, Fairbanks CA, Wang J, Elde R (2001) Developmental shift of vanilloid receptor 1 (VR1) terminals into deeper regions of the superficial dorsal horn: correlation with a shift from TrkA to Ret expression by dorsal root ganglion neurons. Eur J Neurosci 14:293-304.

Holmberg H, Schouenborg J (1996) Postnatal development of the nociceptive withdrawal reflexes in the rat: a behavioural and electromyographic study. J Physiol (Lond) 493:239-252.

Holmberg H, Schouenborg J, Yu YB, Weng HR (1997) Developmental adaptation of rat nociceptive withdrawal reflexes after neonatal tendon transfer. J Neurosci 17:2071-2078.

Huang L, Pallas SL (2001) NMDA antagonists in the superior colliculus prevent developmental plasticity but not visual transmission or map compression. J Neurophysiol 86:1179-1194.

Hughes DI, Scott DT, Todd AJ, Riddell JS (2003) Lack of evidence for sprouting of $A \beta$ afferents into the superficial laminas of the spinal cord dorsal horn after nerve section. J Neurosci 23:9491-9499.

Jackman A, Fitzgerald M (2000) Development of peripheral hindlimb and central spinal cord innervation by subpopulations of dorsal root ganglion cells in the embryonic rat. J Comp Neurol 418:281-298.

Jessell TM (2000) Neuronal specification in the spinal cord: inductive signals and transcriptional codes. Nat Rev Genet 1:20-29.

Katz LC, Shatz CJ (1996) Synaptic activity and the construction of cortical circuits. Science 274:1133-1138.

Knaut H, Blader P, Strahle U, Schier AF (2005) Assembly of trigeminal sensory ganglia by chemokine signaling. Neuron 47:653-666.

Kramer I, Sigrist M, de Nooij JC, Taniuchi I, Jessell TM, Arber S (2006) A role for Runx transcription factor signaling in dorsal root ganglion sensory neuron diversification. Neuron 49:379-393.

Ladle DR, Pecho-Vrieseling E, Arber S (2007) Assembly of motor circuits in the spinal cord: driven to function by genetic and experience-dependent mechanisms. Neuron 56:270-283.

Lee LJ, Lo FS, Erzurumlu RS (2005) NMDA receptor-dependent regulation of axonal and dendritic branching. J Neurosci 25:2304-2311.

Levinsson A, Holmberg H, Broman J, Zhang M, Schouenborg J (2002) Spinal sensorimotor transformation: relation between cutaneous somatotopy and a reflex network. J Neurosci 22:8170-8182.

Lindh B, Aldskogius H, Hokfelt T (1989) Simultaneous immunohistochemical demonstration of intra-axonally transported markers and neuropeptides in the peripheral nervous system of the guinea pig. Histochemistry 92:367-376.

Linkenhoker BA, von der Ohe CG, Knudsen EI (2005) Anatomical traces of juvenile learning in the auditory system of adult barn owls. Nat Neurosci 8:93-98.

Luthi A, Schwyzer L, Mateos JM, Gahwiler BH, McKinney RA (2001) NMDA receptor activation limits the number of synaptic connections during hippocampal development. Nat Neurosci 4:1102-1107.

Meyer MP, Smith SJ (2006) Evidence from in vivo imaging that synaptogenesis guides the growth and branching of axonal arbors by two distinct mechanisms. J Neurosci 26:3604-3614.

Mirnics K, Koerber HR (1995a) Prenatal development of rat primary afferent fibers: I. Peripheral projections. J Comp Neurol 355:589-600.

Mirnics K, Koerber HR (1995b) Prenatal development of rat primary afferent fibers: II. Central projections. J Comp Neurol 355:601-614.

Molander C, Grant G (1985) Cutaneous projections from the rat hindlimb foot to the substantia gelatinosa of the spinal cord studied by transganglionic transport of WGA-HRP conjugate. J Comp Neurol 237:476-484.

Nakatsuka T, Ataka T, Kumamoto E, Tamaki T, Yoshimura M (2000) Alteration in synaptic inputs through $\mathrm{C}$-afferent fibers to substantia gelati- nosa neurons of the rat spinal dorsal horn during postnatal development. Neuroscience 99:549-556.

Oppenheim RW (1991) Cell death during development of the nervous system. Annu Rev Neurosci 14:453-501.

Pang YW, Li JL, Nakamura K, Wu S, Kaneko T, Mizuno N (2006) Expression of vesicular glutamate transporter 1 immunoreactivity in peripheral and central endings of trigeminal mesencephalic nucleus neurons in the rat. J Comp Neurol 498:129-141.

Park JS, Nakatsuka T, Nagata K, Higashi H, Yoshimura M (1999) Reorganization of the primary afferent termination in the rat spinal dorsal horn during post-natal development. Brain Res Dev Brain Res 113:29-36.

Petersson P, Holmer M, Breslin T, Granmo M, Schouenborg J (2001) An imaging system for monitoring receptive field dynamics. J Neurosci Methods 104:123-131.

Petersson P, Waldenstrom A, Fahraeus C, Schouenborg J (2003) Spontaneous muscle twitches during sleep guide spinal self-organization. Nature 424:72-75.

Poo MM (2001) Neurotrophins as synaptic modulators. Nat Rev Neurosci 2:24-32.

Prats-Galino A, Puigdellivol-Sanchez A, Ruano-Gil D, Molander C (1999) Representations of hindlimb digits in rat dorsal root ganglia. J Comp Neurol 408:137-145.

Ralston DD (1994) Cerebellar terminations in the red nucleus of Macaca fascicularis: an electron-microscopic study utilizing the anterograde transport of WGA:HRP. Somatosens Mot Res 11:101-107.

Rethelyi M, Capowski JJ (1977) The terminal arborization pattern of primary afferent fibers in the substantia gelatinosa of the spinal cord in the cat. J Physiol (Paris) 73:269-277.

Rivero-Melian C, Grant G (1990) Distribution of lumbar dorsal root fibers in the lower thoracic and lumbosacral spinal cord of the rat studied with choleragenoid horseradish peroxidase conjugate. J Comp Neurol 299:470-481

Robertson B, Arvidsson J (1985) Transganglionic transport of wheat germ agglutinin-HRP and choleragenoid-HRP in rat trigeminal primary sensory neurons. Brain Res 348:44-51.

Ruthazer ES, Akerman CJ, Cline HT (2003) Control of axon branch dynamics by correlated activity in vivo. Science 301:66-70.

Scheibel ME, Scheibel AB (1968) Terminal axonal patterns in cat spinal cord. II. The dorsal horn. Brain Res 9:32-58.

Schouenborg J (2003) Somatosensory imprinting in spinal reflex modules. J Rehabil Med 35 [Suppl 41]:73-80.

Schouenborg J, Weng HR (1994) Sensorimotor transformation in a spinal motor system. Exp Brain Res 100:170-174.

Schouenborg J, Weng HR, Kalliomaki J, Holmberg H (1995) A survey of spinal dorsal horn neurones encoding the spatial organization of withdrawal reflexes in the rat. Exp Brain Res 106:19-27.

Silos-Santiago I, Jeng B, Snider WD (1995) Sensory afferents show appropriate somatotopy at the earliest stage of projection to dorsal horn. NeuroReport 6:861-865.

Smith AL, Cordery PM, Thompson ID (1995) Manufacture and release characteristics of Elvax polymers containing glutamate receptor antagonists. J Neurosci Methods 60:211-217.

Smith CL (1983) The development and postnatal organization of primary afferent projections to the rat thoracic spinal cord. J Comp Neurol 220:29-43.

Spitzer NC (2002) Activity-dependent neuronal differentiation prior to synapse formation: the functions of calcium transients. J Physiol (Paris) 96:73-80.

Torsney C, Meredith-Middleton J, Fitzgerald M (2000) Neonatal capsaicin treatment prevents the normal postnatal withdrawal of A fibres from lamina II without affecting fos responses to innocuous peripheral stimulation. Brain Res Dev Brain Res 121:55-65.

Waldenstrom A, Thelin J, Thimansson E, Levinsson A, Schouenborg J (2003) Developmental learning in a pain-related system: evidence for a cross-modality mechanism. J Neurosci 23:7719-7725.

West MJ (1999) Stereological methods for estimating the total number of neurons and synapses: issues of precision and bias. Trends Neurosci 22: $51-61$. 